\section{P.L. Miller, R.N. Shiffman, S. Silverstein, S.J. Frawley, P.M. Nadkarni}

Center for Medical Informatics,

Yale University School of Medicine, New Haven CT, USA

\title{
Education and Training
}

\section{Medical Informatics Training at Yale University School of Medicine}

\begin{abstract}
The Yale Center for Medical Informatics has offered Medical Informatics training since 1986. The paper describes the overall structure of the training program, focusing primarily on the postdoctoral fellowship, a major component of which involves an independent project which the fellow carries out under faculty supervision. The paper outlines a number of areas in which such projects have been performed, describes the previous backgrounds and post-training career directions of the trainees, and discusses certain observations based on our experience with the program.
\end{abstract}

\section{The Postdoctoral Fellowship}

The central core of the fellowship training involves each fellow identifying one or two projects which can be carried out independently, under faculty supervision. The emphasis is on: (1) defining an interesting project that can be carried out in a reasonable timeframe, (2) carrying out the project, which typically involves a significant amount of computer programming, (3) periodically presenting the status of the project in a working seminar to faculty and fellows, (4) writing up one or more papers describing the project, and (5) presenting the work at one or more national conferences.

A fellow's project often builds on some aspect of an ongoing YCMI research project. In this case, it is important to define the fellow's project so that it is not in the critical path of the broader project, allowing the fellow to avoid pressures that would be inappropriate for a training experience. Other fellows have particular interests that lead them to pursue a project that is unrelated to ongoing activities within the YCMI. To accommodate such an interest, it is often necessary for a YCMI faculty member to collaborate with a faculty member in some other field to supervise the fellow's project. The overall goal is to help the fellows pursue projects that suit their unique backgrounds and interests and which lead in directions which they will want to build upon in their later careers.

In additional to this central experience of carrying out an independent Medical Informatics project, fellows become involved in a number of other activities:

1. Fellows attend a weekly teaching seminar which over a two-year period covers a breadth of Medical Informatics topics. The goal is to provide a comprehensive overview of the field of Medical Informatics as a whole. A variety of formal lectures are also offered.

2. Fellows are encouraged to attend any relevant courses offered at Yale, for example, in computer science, epidemiology and public health, or graphic design. These courses are 
usually, but not always, attended on a non-credit basis. The selection of courses is tailored to each fellow's background and interests.

3. Ongoing YCMI projects typically involve a weekly group meeting of the participants, where the status of the project is discussed, any problems are identified, and potential solutions and future plans are discussed. Fellows are encouraged to attend these weekly meetings for one or more projects in which they are not directly involved. This experience helps provide the fellow with a broader perspective on how interdisciplinary Medical Informatics research is performed.

4. YCMI faculty and others closely affiliated with the Center are involved in a variety of institutional computing initiatives within the School and within the medical center as a whole. Fellows are encouraged to participate in these institutional computing activities, which givethemexperienceinhelping carry out applied computing projects within an academic medical center.

5. Fellows are encouraged to assist in the YCMI's teaching activities to give them experience in presenting Medical Informatics material in a didactic setting.

6. Although no patient care is required, we encourage each fellow to spend up to one day a week in clinical activities. This arrangement helps maintain clinical skills, can let the fellow feel part of Yale on a clinical level, and can serve as the context for developing project ideas and productive clinical collaborations.

The postdoctoral fellowship usually lasts two years. It is occasionally extended to include a third year when a fellow requests additional time to explore an interesting project more fully.

\section{Representative Project Areas}

This section describes several current and recent areas in which fellows have performed Medical Informatics projects. Representative publications are included as references.

\section{Clinical Decision Support}

Alongstanding projecthas involved the development of clinical advicegiving programs, with a particular focus on exploring the critiquing approach to bringing computer-based advice to the practicing clinician [1]. A recent emphasis has been on computer-based knowledge processing for clinical practice guidelines [2]. Guidelines can serve as valuable knowledge sources for systems that assist clinical decision-making. This project explores the acquisition and representation of guideline knowledge as rule sets. Previous activities have focused on defining methods for translation of guideline prose and flowcharts into rule sets, logical verification of rule sets, and simplification of comprehensive rule bases.

\section{Linking Medicine to the World of Network-Based Information}

This project started as part of the multi-institutional Unified Medical Language System (UMLS) project of the National Library of Medicine [3]. It has included building NetMenu, a "network menu" that resides on a desktop Macintosh or PC and helps the user connect easily to network-based information resources located anywhere in the world. The project has also developed an "information sources map" (ISM) to provide active assistance to help determine which of many potential information resources on the network might be relevant to a particular question [4].
An Intelligent Radiologic Workstation

This collaboration involves a set of projects aimed towards developing an intelligent radiologic workstation. The projects combine research developing expert consultation systems for radiology and research exploring the intelligent retrieval of radiologic images. This project has focused on several domains:(1) pulmonary complications of lymphoproliferative disease, (2) breast cancer as seen on mammograms, and (3) diagnostic ultrasound [5,6].

\section{Linking from Psychiatric Patient Records to Information Retrieval}

This project(PsychTopix) explored how a conceptual outline of clinical topics in psychiatry can help guide a clinician from an online psychiatric consultation report to relevant bibliographic retrieval. The approach also helps guide the clinician to other relevant online information, such as 1) relevant previous cases, 2) textual information, and 3) expert consultation systems [7].

\section{Integrated Access to Multiple Databases}

This project built a prototype system to explore the ability of the computer to assist the clinician in occupational medicine, focusing on the problem of occupational asthma. The project explored how the computer can provide clinical decision support using 1) expert knowledge of the domain combined with 2) the ability to retrieve information from multiple online databases in an integrated fashion [8].

\section{Computer-Assisted Instruction \\ Using Clinical Images}

The Yale Center for Advanced Instructional Media, directed by Dr. Carl Jaffe, is engaged in a set of long-term projects to develop computer-aided instruction environments for medical students at Yale, and also on a multiuniversity level. Dr. Jaffe has collabo- 
rated with various faculty from Diagnostic Radiology and Cardiology to develop teaching programs involving both static and dynamic images, including: (1) cardiac echo, (2) cardiac MRI, (3) cardiac nuclide imaging, and (4) cardiac catheterization. Projects incorporate computer-aided instruction techniques to assess learning accomplishment. The software also records user audit trails which can be used to gain better understanding of learning strategies for various medically important topics such as imagebased understanding [9].

\section{The Human Genome Project and the Human Brain Project}

The YCMI is involved in two projects which provide Internet-based informatics support to inter-institutional biomedical research collaborations [10]. One is a collaborative gene mapping center involving laboratories at Yale and at the Albert Einstein College of Medicine. The other is a neuroscience collaboration involving laboratories at Yale and at several other institutions. Providing such informatics support involves first understanding the scientific field in considerable detail, and then helping provide network connectivity including a shared database in which experimental results can be entered and analyzed, and also developing informatics tools to help the scientists better understand their data and the underlying phenomena being studied.

\section{Knowledge-Based Image Processing}

The Yale Image Processing and Analysis Group, directed by Prof. James Duncan, is developing strategies to use information derived from biomedical images for: (1) improved understanding of basic anatomical and physiological relationships in normal and disease states, and (2) more accurate and reproducible clinical diagnosis. Current projects include: (1) automated segmentation of the left ven- tricle of the heart from 4D cine Magnetic Resonance images (MRI) and of the temporal lobes of the brain from static 3D MR images [11], (2) tracking and modeling nonrigid motion of the heart using shape-based strategies and dense field MR phase velocity data [12], and (3) indexing medical image databases according to information derived mathematically from the images.

\section{Parallel Computation in Biomedicine}

This project applied parallel computation in a number of areas in molecular biology (biologic sequence analysis, genetic linkage analysis, and molecular dynamics) and clinical medicine (intelligent cardiovascular monitoring) [13], using the machineindependent parallel programming language, Linda, developed at Yale by Prof. David Gelernter. To run on a parallel computer, a program must be broken up into pieces which can be executed simultaneously on different computer processors, thereby speeding up the program as a whole [14].

\section{Previous Background of the Trainees}

Most clinical fellows have completed residency, in diverse specialties. One fellow had only completed a year of internship. A second fellow had completed internship and one year of a residency which he had decided to leave. The backgrounds of our postdoctoral fellows (MDs and PhDs) were:

Anesthesiology (1), Diagnostic Radiology (2), Internal Medicine (5), Laboratory Medicine (3), Neurology (1), OB/Gyn(1), Preventive Medicine (1), Psychiatry (1), Internship only (1), Partial residency (1), Biology (1), Genetics (1).

Since Yale does not have a degree program in Medical Informatics, we support Ph.D. candidates already en- rolled at Yale in a variety of departments, who are working on Medical Informatics-related dissertations. The training program of these trainees is largely determined by their home department. Predoctoral trainees came from Computer Science (3), Electrical Engineering (3), and Genetics (1).

\section{Post-Training Career Directions}

Our fellows have shown a strong tendency to pursue academic careers after completing the fellowship. Most clinical fellows have found academic homes in their clinical specialties, although three have remained at the YCMI in positions where Medical Informatics was their primary responsibility. Of the MDs or MDs/PhDs, five became academic faculty (clinical departments), three became academic faculty (medical informatics). One went to pharmaceutical industry, and one entered a clinical fellowship. One $\mathrm{PhD}$ became academic faculty, and another went to biotechnological industry.

The predoctoral trainees went to academic faculty (2), industry (computing) (1), or are still finishing their training.

\section{Observations}

This section highlights several observations based on our experience with the training program.

\section{How much Computer Experience is Required of an Entering Fellow?}

For an entering postdoctoral fellow, we require at a minimum some experience in computer programming, either from formal programming courses or from significant practical experience. Without this background, we feel that fellows would not be prepared to embark upon the type of 
Medical Informatics projects that we would like them to perform. Also, without some programming experience, we feel that fellows have not been able to "test reality" enough to commit a major part of their career to Medical Informatics.

\section{How Best to Combine Training in Informatics for Clinical Medicine and the Biosciences}

Although the YCMI has major ongoing informatics projects in both clinical medicine and the biosciences, most of our postdoctoral fellows have been from the clinical domain. As a result, we have not had to develop a general approach for integrating informatics training in these two areas. Instead, we have dealt with each bioscience fellow on an individual basis, and tailored their interaction with the clinical informatics training activities to their background and interests. In the future, if we have a significant number of fellows in both the clinical and bioscience areas, we will need to confront how best to combine their training. Our general feeling is that the research issues and underlying domain knowledge are quite different in the two areas, but that both types of fellow can benefit from interaction and exposure to both these two sides of biomedical informatics. The best way to achieve this will likely be through a mixture of parallel training activities and joint training activities.

\section{Fellows often Help Incubate}

Promising Research Collaborations

We have found that the fellowship has had a number of institutional benefits. One benefit is that the fellow often serves as a vehicle through which a research collaboration gets established between YCMI faculty and faculty in other disciplines. As both faculty members meet on a regular basis to supervise the fellow's project, a research direction evolves which can then develop into a robust collabora- tion that grows in scope.

\section{The Training Program is a Valuable Source of Faculty to Help \\ Strengthen our Institution}

Another benefit is that the training program has served as a source of faculty who can help strengthen Medical Informatics activities within our institution. For example, projects and activities undertaken during the fellowship often evolve to become longer term projects which help provide a foundation for a fellow's early career as a junior faculty member. Depending on the fellow, these activities may involve Medical Informatics research or may involve performing an institutional computing role within our medical center.

Acknowledgment: These training activities were supported in part by NIH grant T15 LM07056 from the National Library of Medicine.

\section{References}

1. Miller PL. Expert Critiquing Systems. New York: Springer-Verlag, 1986.

2. Shiffman RN, Greenes RA Improving clinical guidelines with logic and decision table techniques: Application to hepatitis immunization recommendations. Med Decis Making 1994; 14:145-54.

3. Clyman JI, Powsner SM, Paton JA, Miller PL. Using the UMLS information sources map to access online reference materials. Bull Med Libr Assoc 1993;8:207-216.

4. Miller PL, Frawley SJ, Wright L, Roderer NK, Powsner SM. Lessons learned from a pilot implementation of the UMLS information sources map. J Am Med Inform Assoc 1995;2:102-15.

5. Swett HA, Fisher PR, Miller PL, Mutalik PG. Expert system controlled image display. Radiology 1989;172:487-93.

6. Cohn AI, Fisher PR, Mutalik P, Miller PL, Swett HA. AXON: Knowledge-based retrieval of radiographic studies using "axes" of clinical relevance. Comput Biomed Res 1990;23:199-221.

7. Powsner SM, Miller PL. Automated online transition from the medical record to the psychiatric literature. Meth Inform Med 1992;31:169-74.

8. Berman L, Cullen MR, Miller PL. Auto- mated integration of external databases: A knowledge-based approach to enhancing rule-based expert systems. Comput Biomed Res 1993;26:230-41.

9. Jaffe C, Lynch P. Hypermedia for medical imaging instruction. Radiology 1989; 171:475-80.

10. Miller PL, Nadkarni PM, Kucherlapati R, Krauter KS, Kidd KK, Ward DC, Shepherd GM, Berkowicz D. Network-based informatics support of research collaboration in the Human Genome Project and the Human Brain Project. In: Greenes RA, Peterson HE, Protti DJ, eds. Proceedings $M E D I N F O$ 95. Amsterdam: North-Holland, 1995: 1541-4.

11. Staib LH, Duncan JS. Boundary finding with parametrically deformable models. IEEE T Pattern Anal 1992;14:1061-75.

12. McEachen J, Meyer F, Constable RT, Nehorai A, Duncan J. A recursive filter for phase velocity assisted, shape-based tracking of cardiac nonrigid motion. In: Grimson E, ed. Proc. Fifth International Conference on Computer Vision. Los Alamitos, CA: IEEE Computer Society Press, 1995: 653-8.

13. Sittig DF, Shifman MA, Nadkarni P, Miller PL. Parallel computation for medicine and biology: Experience with Linda at Yale University. Int J Supercomput Ap 1992;6:147-63.

14. Miller PL, Nadkarni PN, BercovitzP. Harnessing networked workstations as a powerful parallel machine: A general paradigm illustrated using three programs for genetic linkage analysis. Comput Appl Biosci 1992;8:141-7.

Address of the first author:

Perry L. Miller, MD, PhD,

Center for Medical Informatics,

333 Cedar Street,

P.O. Box 208009,

New Haven, CT 06520-8009,

USA.

E-mail: perry.miller@yale.edu 